\title{
Front Matter: Volume 10061
}

, "Front Matter: Volume 10061," Proc. SPIE 10061, Microfluidics, BioMEMS, and Medical Microsystems XV, 1006101 (18 April 2017); doi:

10.1117/12.2275886

SPIE. Event: SPIE BiOS, 2017, San Francisco, California, United States 


\title{
PROGRESS IN BIOMEDICAL OPTICS AND IMAGING
}

\section{Microfluidics, BioMEMS, and Medical Microsystems XV}

\author{
Bonnie L. Gray \\ Holger Becker \\ Editors
}

28-30 January 2017

San Francisco, California, United States

Sponsored by

SPIE

Co-sponsored by

microfluidic ChipShop GmbH (Germany)

The Ohio Center for Microfluidic Innovation at the University of Cincinnati (United States)

Published by

SPIE 
The papers in this volume were part of the technical conference cited on the cover and title page. Papers were selected and subject to review by the editors and conference program committee. Some conference presentations may not be available for publication. Additional papers and presentation recordings may be available online in the SPIE Digital Library at SPIEDigitallibrary.org.

The papers reflect the work and thoughts of the authors and are published herein as submitted. The publisher is not responsible for the validity of the information or for any outcomes resulting from reliance thereon.

Please use the following format to cite material from these proceedings:

Author(s), "Title of Paper," in Microfluidics, BioMEMS, and Medical Microsystems XV, edited by Bonnie L. Gray, Holger Becker, Proceedings of SPIE Vol. 10061 (SPIE, Bellingham, WA, 2017) Seven-digit Article CID Number.

ISSN: 1605-7422

ISSN: 2410-9045 (electronic)

ISBN: 9781510605633

ISBN: 9781510605640 (electronic)

Published by

SPIE

P.O. Box 10, Bellingham, Washington 98227-0010 USA

Telephone +1 3606763290 (Pacific Time) · Fax +1 3606471445

SPIE.org

Copyright (c) 2017, Society of Photo-Optical Instrumentation Engineers.

Copying of material in this book for internal or personal use, or for the internal or personal use of specific clients, beyond the fair use provisions granted by the U.S. Copyright Law is authorized by SPIE subject to payment of copying fees. The Transactional Reporting Service base fee for this volume is $\$ 18.00$ per article (or portion thereof), which should be paid directly to the Copyright Clearance Center (CCC), 222 Rosewood Drive, Danvers, MA 01923. Payment may also be made electronically through CCC Online at copyright.com. Other copying for republication, resale, advertising or promotion, or any form of systematic or multiple reproduction of any material in this book is prohibited except with permission in writing from the publisher. The CCC fee code is $1605-7422 / 17 / \$ 18.00$.

Printed in the United States of America.

Publication of record for individual papers is online in the SPIE Digital Library.

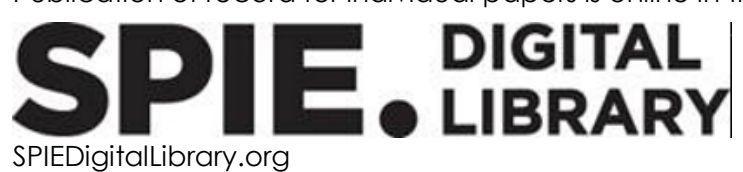

Paper Numbering: Proceedings of SPIE follow an e-First publication model. A unique citation identifier (CID) number is assigned to each article at the time of publication. Utilization of CIDs allows articles to be fully citable as soon as they are published online, and connects the same identifier to all online and print versions of the publication. SPIE uses a seven-digit CID article numbering system structured as follows:

- The first five digits correspond to the SPIE volume number.

- The last two digits indicate publication order within the volume using a Base 36 numbering system employing both numerals and letters. These two-number sets start with $00,01,02,03,04$, $05,06,07,08,09,0 A, 0 B \ldots$ OZ, followed by 10-1Z, 20-2Z, etc. The CID Number appears on each page of the manuscript. 


\title{
Contents
}

\author{
vii Authors \\ ix Conference Committee
}

\section{SESSION 1 MANUFACTURING I}

1006102 Hybrid carbon nanotube-polymer scaffolds for cardiac tissue regeneration (Invited Paper) [10061-1]

1006104 Rapid structuring of proteins on filter paper using lithography [10061-3]

1006105 Graphene doped ZnO films for photoelectrowetting on microchannels [10061-4]

\section{SESSION 2 MICROFLUIDIC DEVICES I}

1006108 Investigation of the capillary flow through open surface microfluidic structures [10061-8]

10061 OA AC electrothermal technique in microchannels [10061-10]

SESSION 3 MANUFACTURING II

10061 OD Fast and cheap fabrication of molding tools for polymer replication [10061-14]

SESSION 4 APPLICATIONS I

10061 OE 3D printed disposable optics and lab-on-a-chip devices for chemical sensing with cell phones (Invited Paper) [10061-15]

10061 OF Implementation of a protocol for assembling DNA in a Teflon tube [10061-16]

$10061 \mathrm{OH}$ Towards rapid prototyped convective microfluidic DNA amplification platform [10061-18]

\section{SESSION 5 OPTOFLUIDICS I}

10061 Ol System-level integration of active silicon photonic biosensors (Invited Paper) [10061-19]

10061 0J Light field 3D endoscope based on electro-wetting lens array [10061-20]

10061 OL An optofluidic approach for gold nanoprobes based-cancer theranostics [10061-22] 
10061 OM A portable fluorescent sensing system using multiple LEDs (Invited Paper) [10061-23]

1006100 Chemiluminescence generation and detection in a capillary-driven microfluidic chip [10061-25]

10061 OP Multipath trapping dynamics of nanoparticles towards an integrated waveguide with a high index contrast [10061-26]

\section{SESSION 7 MICROFLUIDIC DEVICES II}

10061 OS Piezoelectric micromachined ultrasonic transducers and micropumps: from design to optomicrofluidic applications [10061-29]

10061 OT Optimized AC electrothermal micromixing design for biofluid systems [10061-30]

\section{SESSION $8 \quad$ APPLICATIONS II}

$100610 X \quad$ Microfluidic separation of particles from whole blood using shear induced diffusion [10061-34]

$100610 Y$ Controllable gas in oil in water double emulsion formation in a non-planar microfluidic device [10061-35]

\section{SESSION 9 APPLICATIONS III}

$100610 Z$ Peptide library synthesis on spectrally encoded beads for multiplexed protein/peptide bioassays (Invited Paper) [10061-36]

$1006110 \quad$ Microfluidic system for in-vitro hypoxia assays [10061-37]

1006111 Lab-on-a-chip plafform for high throughput drug discovery with DNA-encoded chemical libraries [10061-38]

1006112 Thermally assisted acoustophoresis as a new stiffness-based separation method (Best Student Paper) [10061-39]

\section{SESSION 10 MEDICAL DEVICES}

1006113 Integration of systems biology with organs-on-chips to humanize therapeutic development (Invited Paper) [10061-40]

1006116 Microfluidic devices for stem-cell cultivation, differentiation and toxicity testing [10061-43] 
POSTER SESSION

1006119 Liquid-phase reduction synthesis of mono-dispersed gold nanoparticles on glass microfluidic device with flow rate control [10061-44]

10061 1A Numerical study of insulator-based dielectrophoresis method for circulating tumor cell separation [10061-47]

Proc. of SPIE Vol. $100611006101-5$

Downloaded From: https://www.spiedigitallibrary.org/conference-proceedings-of-spie on 25 Apr 2023 Terms of Use: https://www.spiedigitallibrary.org/terms-of-use 
Proc. of SPIE Vol. 10061 1006101-6

Downloaded From: https://www.spiedigitallibrary.org/conference-proceedings-of-spie on 25 Apr 2023 Terms of Use: https://www.spiedigitallibrary.org/terms-of-use 


\section{Authors}

Numbers in the index correspond to the last two digits of the seven-digit citation identifier (CID) article numbering system used in Proceedings of SPIE. The first five digits reflect the volume number. Base 36 numbering is employed for the last two digits and indicates the order of articles within the volume. Numbers start with 00, 01, 02, 03, 04, 05, 06, 07, 08, 09, OA, OB...0Z, followed by 10-12, 20-2Z, etc.

Aghaamoo, Mohammad, 1A

Aghilinejad, Arian, 1A

Ahadian, Samad, 02

Ajit, Smrithi, $\mathrm{OH}$

Al-Aribe, Khaled, 05

Al'Mrayat, O., 01

Barnett, Jonathan Z., OM

Baxter, Brian, $\mathrm{OZ}$

Becker, Holger, 16

Brower, Kara, $\mathrm{OZ}$

Busek, M., 10, 11

Chen, Wen Li Kelly, 13

Chen, Xiaolin, IA

Cheung, K., Ol

Choi, Jin-Woo, OM

Chrostowski, L., OI

Cirit, Murat, 13

Clark, Amanda M., 13

Comina, G., OE

Cook, Peter R., OF

Cui, M., 11

Dalton, Colin, OA, OT

Dattner, Y., Ol

Dave, Abhishek, $\mathrm{OH}$

Davenport-Huyer, Locke, 02

Delamarche, Emmanuel, 00

Dolatmoradi, Ata, 12

Edington, Collin D., 13

El-Zahab, Bilal, 12

Fang, C., Ol

Fang, Yifeng, $\mathrm{OX}$

Feuerborn, Alexander, OF

Filippini, D., OE

Fiorini, Paolo, 08

Fordyce, Polly M., $0 z$

Gärtner, Claudia, 16

Goel, Sanket, $\mathrm{OH}$

Griffith, Linda G., 13

Günzner, S., 10, 11

Gutierrez-Wing, M. Teresa, OM

Hansen-Hagge, Thomas, 16

Harink, Björn, $\mathrm{OZ}$

Helmer, Dorothea, 04

Huang, Bobo, $\mathrm{OX}$

Huang, Cong, OY

Jones, Benjamin, 08

Jung, Gyu Suk, OJ

Keller, Nico, 04

Khazaaleh, Shadi, OS
Kimerling, Lionel C., OP

Kirschner, Nadine, OD

Klotzbach, U., 10, 11

Knopf, George K., 05

Koch, Katharina K. C., OY

Kotz, Frederik, 04

Kurtz, Andreas, 16

Lagae, Liesbet, 08

Laplatine, L., 0 I

Lee, Jin Su, OJ

Li, Guifang, OP

Liang, Xiao, OX

Liang, Yitao, $0 \mathrm{X}$

Luan, E., Ol

Madzik, Mateusz T., OS

Michel, Jurgen, OP

Mohan, K. N., OH

Mrowka, Ralf, 16

Nargang, Tobias M., 04

Navi, Maryam, OA, OT

Nguyen, Huy Q., OZ

Panwar, Nishtha, OL

Papautsky, Ian, OX

Praveen, Hemanth Mithun, $\mathrm{OH}$

Radisic, Milica, 02

Ramon, Charlotte, 00

Rapp, Bastian E., 04, OD

Ratner, D. M., 01

Reddavide, F. V., 11

Rezaiezadeh, S., 01

Richter, Christiane, OD

Rusch, Kelly A., OM

S. B., Puneeth, $\mathrm{OH}$

Saeed, Numan, OS

Salari, Alinaghi, OA, OT

Schmieder, F., 10

Sesham, Bharat, $\mathrm{OH}$

Shin, Young-Ho, OM

Smith, Nathaniel, 02

Song, Peiyi, OL

Sonntag, F., 10, 11

Steege, T., 10

Steinfelder, C., 10, 11

Suska, A., OE

Taha, Inas, OS

Taher, Ahmed, 08

Tanabe, Yu, 19

Temiz, Yuksel, 00

Thorn, Kurt S., $\mathrm{OZ}$ 
Tian, Hao, OP

Tjin, Swee Chuan, OL

Trumper, David L., 13

Tu, Chunlong, $\mathrm{OX}$

Viegas, Jaime, OS

Walsh, Edmond J., OF

Wells, Alan, 13

Wölfl, Stefan, 16

Won, Yong Hyub, 0J

Worgull, Matthias, OD

Yagyu, Hiromasa, 19

Yang, Chengbin, $\mathrm{OL}$

Ye, Xuesong, $\mathrm{OX}$

Yin, Rui-Xue, OY

Yong, Ken-Tye, OL

Zhang, Hong-Bo, OY

Zhang, Lin, OP

Zhang, Wen-Jun, OY

Zhang, Y., 11

Zhou, Jian, OX 


\section{Conference Committee}

Symposium Chairs

James G. Fujimoto, Massachusetts Institute of Technology

(United States)

R. Rox Anderson, Wellman Center for Photomedicine, Massachusetts General Hospital (United States) and Harvard School of Medicine (United States)

Program Track Chairs

Tuan Vo Dinh, Fitzpatrick Institute for Photonics, Duke University (United States)

Anita Mahadevan-Jansen, Vanderbilt University (United States)

Conference Chairs

Bonnie L. Gray, Simon Fraser University (Canada)

Holger Becker, microfluidic ChipShop GmbH (Germany)

Conference Program Committee

Brian W. Anthony, Massachusetts Institute of Technology (United States)

Yolanda Fintschenko, LabSmith, Inc. (United States)

Bruce K. Gale, The University of Utah (United States)

Albert K. Henning, Aquarian Microsystems (United States)

Yu-Cheng Lin, National Cheng Kung University (Taiwan)

Yuehe Lin, Pacific Northwest National Laboratory (United States)

Ciara K. O'Sullivan, Universitat Rovira i Virgili (Spain)

Ian Papautsky, University of Cincinnati (United States)

Bastian E. Rapp, Karlsruher Institut für Technologie (Germany)

Thomas Stieglitz, Albert-Ludwigs-Universität Freiburg (Germany)

Sindy Kam-Yan Tang, Stanford University (United States)

Albert van den Berg, MESA+ Institute for Nanotechnology

(Netherlands)

Wanjun Wang, Louisiana State University (United States)

Bernhard H. Weigl, PATH (United States) 
Session Chairs

1 Manufacturing I

Bonnie L. Gray, Simon Fraser University (Canada)

Holger Becker, microfluidic ChipShop GmbH (Germany)

2 Microfluidic Devices I

Bonnie L. Gray, Simon Fraser University (Canada)

3 Manufacturing II

Samad Ahadian, University of Toronto (Canada)

4 Applications I

Jin Woo Choi, Lovisiana State University (United States)

5 Optofluidics I

Daniel Filippini, Linköping University (Sweden)

6 Optofluidics II

Loic Laplatine, The University of British Columbia (Canada)

7 Microfluidic Devices II

Jian Zhou, Zhejiang University (China)

8 Applications II

Polly Fordyce, Stanford University (United States)

9 Applications III

Somin Eunice Lee, University of Michigan (United States)

10 Medical Devices

Bonnie L. Gray, Simon Fraser University (Canada)

Holger Becker, microfluidic ChipShop GmbH (Germany) 